VOL. 38 (1988) [141-150]

\title{
FINITE COVERINGS BY 2-ENGEL GROUPS
}

\section{LUISE-ChaRlotTe KaPPE}

\begin{abstract}
Baer's characterisation of central-by-finite groups as groups possessing a finite covering by abelian subgroups is the starting point for this investigation. We characterise groups with a finite covering by 2-Engel subgroups as groups for which the subgroup of right 2-Engel elements has finite index; and the groups having a finite covering by normal 2Engel subgroups are exactly the 3-Engel groups among those having a finite covering by 2-Engel subgroups. The second centre of a group having a finite covering by class two subgroups does not necessarily have finite index. However, a group has a finite covering by subgroups in a variety containing all cyclic groups if the margin of this variety in the group has finite index.
\end{abstract}

\section{INTRODUCTION AND RESULTS}

Bernhard Neumann [7],suggested the following problem:

Given a group $G$ covered by finitely many subgroups $H_{1}, H_{2}, \ldots, H_{n}$ with intersection $D$. If $H_{1}, H_{2}, \ldots, H_{n}$ possess a certain property $\mathcal{E}$, what can be said about $D$ in relation to $G$, or about $G$ itself?

His attention was drawn to this problem by the characterisation of central-by-finite groups given by Reinhold Baer:

Theorem A. ([7, 9, 4.16]) A group is central-by-finite if and only if it has a finite covering consisting of abelian subgroups.

In [7, Section 6], B.H. Neumann raises the question as to whether Baer's result can be extended to finite coverings by Engel-groups and $k$-Engel groups. To give an affirmative answer in the case of finite coverings by 2-Engel subgroups is the main topic of this paper.

We define $\varepsilon_{k}(x, y)=[x, k y]=[[x, k-1, y], y]$ as the $k$-Engel word, where $\varepsilon_{1}(x, y)=$ $[x, 1 y]=[x, y]$ is the commutator of $x$ and $y$. An element $a$ in a group $G$ is a right $k$-Engel element if $[a, k]=1$ for all $x$ in $G$. A group is a $k$-Engel group if $[x, k y]=1$ for all $x, y \in G$. Let $G$ be a group and let

$$
L(G)=\{a \in G ; \forall x \in G[a, 2 x]=1\}
$$

be the set of right 2-Engel elements. In [4], W. Kappe has shown that $L\left(C^{\prime}\right)$ is a characteristic subgroup of $G$. For finite coverings by 2 -Engel groups we can now formulate our result.

Received 30 October 1987

Copyright Clearance Centre, Inc. Serial-fee code: 0004-9729/88 \$A2.00+0.00. 
Theonem 1. A group $G$ is the union of finitely many 2-Engel subgroups if and only if $G / L(G)$ is finite.

It remains an open question in which way Baer's characterisation can be extended to finite coverings by $k$-Engel groups, particularly in light of the fact that for $k \geqslant 3$ the set of right $k$-Engel elenents does not necessarily form a subgroup (see [5]).

In [1], Theorem $\mathrm{A}$ was extended to the case of finite coverings by abelian normal subgroups as follows.

THEOREM B. ([1, Theorem 2]) A group $G$ is the union of finitely many abelian normal subgroups if and only if $G / Z(G)$ is finite and $G$ is a 2-Engel group.

A direct analogue for finite coverings by normal 2-Engel subgroups is the following theorem.

THEOREM 2. A group $G$ is the union of finitely many normal 2-Engel subgroups if and only if $G / L(G)$ is finite and $G$ is a 3-Engel group.

This theorem is based on Theorem 1, a characterisation of 3-Engel groups given in [3], and a corollary which is in fact another characterisation of 3-Engel groups. (See Theorem $\mathbf{E}$ and Corollary 6.) Theorem A brings to mind a familiar result which is essentially due to Schur [10]. For other sources we refer to [9, page 102].

Theorem C. If $G$ is a group whose centre has index $n$, then $G^{\prime}$ is finite and $\left(G^{\prime}\right)^{n}=1$.

In light of this result and Theorem 1 , we may ask whether the finiteness of $G / L(G)$ implies that the word subgroup $\varepsilon_{2}(G)=\langle[x, 2 y] ; x, y \in G\rangle$ is finite. However, this is not the case. In the last section a group $H$ is constructed with $H / L(H)$ finite but $\varepsilon_{2}(H)$ is not finite (Proposition 8 , (iv) and (viii)).

In this context we may raise the question as to whether the $k$-th centre has finite index in a group if the group has a finite covering by subgroups of nilpotency class $k$. The question has a negative answer, as shown by the same counterexample. The group $H$ has a finite covering by subgroups of class 2 , but $Z_{2}(H)$ does not have finite index in $H$ (Proposition 8, (v) and (vi)).

It is however true that the finite index of the $k$-th centre implies that the group has a finite covering by subgroups of class $k$. This is a consequence of our next result, relating the finite index of a suitable margin to the existence of a finite covering by subgroups in a variety $\mathcal{V}$.

The margin of a word was introduced by $P$. Hall in [2]. Let $\psi\left(x_{1}, \ldots, x_{n}\right)$ be a word in the variables $x_{1}, \ldots, x_{n}$. The $i$-th partial margin of $\psi$ in a group $G$ is defined 
as

$$
\psi_{i}^{*}(G)=\left\{a \in G ; \psi\left(g_{1}, \ldots, a g_{i}, \ldots, g_{n}\right)=\psi\left(g_{1}, \ldots, g_{i}, \ldots, g_{n}\right) \text { for } g_{1}, \ldots, g_{n} \in G\right\} .
$$

The margin of $\psi$ in $G$ is defined as

$$
\psi^{*}(G)=\bigcap_{i=1}^{n} \psi_{i}^{*}(G) .
$$

The partial margins, and thus the margin of a word in a group $G$, are characteristic subgroups in $G$.

Let $V$ be a set of words and $\mathcal{V}$ the variety of groups defined by the set $V$. Consider

$$
\psi_{V}^{*}(G)=\bigcap_{\psi \in V} \psi^{*}(G)
$$

We say that $\psi_{V}^{*}(G)$ is the margin of the variety $\mathcal{V}$ in $G$, Obviously $\psi_{V}^{*}(G)$ is in $\mathcal{V}$.

The following result holds for a large class of words, the class of all commutator words (see [8, page 4]).

Theorem 3. Let $V$ be a set of words and let $G$ be a group. Assume that the variety $\mathcal{V}$ defined by $V$ contains all cyclic groups. Then $G / \psi_{V}^{*}(G)$ finite implies that $G$ has a finite covering by subgroups in $\mathcal{V}$.

In general, the finiteness of $G / \psi_{V}^{*}(G)$ does not imply that $G$ has a finite covering by subgroups in $\mathcal{V}$, as the following example shows. Let $\psi(x)=x^{2}$. It can be shown easily that for any group $G$

$$
\psi^{*}(G)=\left\{a \in Z(G) ; a^{2}=1\right\}
$$

Consider $G=F \times E_{2}$, where $F$ is a finite group of odd order greater than one, and $E_{2}$ an elementary abelian 2-group which is not necessarily finite. Now $\psi^{*}(G)=$ $E_{2}$, and $G / E_{2}=F$ is finite. But $G$ is not the union of finitely many elementary abelian 2-groups. Observing that $\gamma_{k}\left(x_{1}, \ldots, x_{k}\right)=\left[x_{1}, \ldots, x_{k}\right]$, the lower central word of weight $k$, and the $k$-Engel word $\varepsilon_{k}(x, y)$ are commutator words, and that the respective margins in a group $G$ are $Z_{k-1}(G)$ (see [2]) and $\varepsilon_{k}^{*}(G)$, we obtain the following corollary.

Corollary 4. Let $G$ be a group with $G / Z_{k}(G)$ or $G / \varepsilon_{k}^{*}(G)$ finite. Then $G$ has a finite covering by subgroups of nilpotency class $k$, or by $k$-Engel subgroups, respectively.

The converse of Theorem 3, that is, that the existence of a finite covering of a group by subgroups in a variety $\mathcal{V}$ which contains all cyclic groups implies the finiteness of 
the index of $\psi_{V}^{*}(G)$, is rarely true. As already mentioned, the counterexample $H$ has a finite covering by class 2 subgroups, however $Z_{2}(H)$ does not have finite index. In addition, $\varepsilon_{2}^{*}(H)$, the 2-E்ngel margin of $H$, does not have finite index, but $H$ has a finite covering by 2-Engel subgroups (Proposition 8, (iv) and (vi)).

\section{Some Preliminary Steps}

The following result of Neumann is basic in the investigation of finite coverings of groups by subgroups. It allows us to assume that all subgroups in a finite covering of a group have finite index.

Theorem D. ([6, 4.4]) Let $G=\bigcup_{i=1}^{n} H_{i} g_{i}$ where $H_{1}, \ldots, H_{n}$ are (not necessarily distinct) subgroups of $G$. Then if we omit from the union any coset $H_{i} g_{i}$ for which $\left[G: H_{i}\right]$ is infinite, the union of the remaining cosets is still all of $G$.

The next lemma collects essential facts about 2-Engel elements needed throughout this paper.

Lemma 5. ([4]) Let $a \in L(G)$. Then:

(i) $\left[x,{ }_{2} a\right]=1$ for all $x \in G$;

(ii) $[[x, y],[x, a]]=1$ for all $x, y \in G$;

(iii) $\left[x, a^{k}\right]=\left[x^{k}, a\right]=[x, a]^{k}$ for all $x \in G, k \in Z$.

Denote by $\left\langle x^{G}\right\rangle$ the normal closure of an element $x$ in $G$, and let $c(G)$ denote the nilpotency class of a group $G$. The following characterisation of 3-Engel groups was given earlier by W. Kappe and the author.

Theorem E. ([3]) The following conditions on a group $G$ are equivalent:

(i) $c\left(\left\langle x^{G}\right\rangle\right) \leqslant 2$ for all $x \in G$;

(ii) $\left\langle x^{G}\right\rangle$ is a 2 -Engel group for all $x \in G$;

(iii) $G$ is a 3-Engel group.

Here we prove the following corollary.

Corollary 6. A group $G$ is a 3-Engel group if and only if $\left\langle x^{G}\right\rangle L(G)$ is a 2-Engel group for all $x \in G$.

Proof: It suffices to show that for any 3-Engel group $G$ the normal subgroup $\left\langle x^{G}\right\rangle L(G)$ is a 2-Engel group for all $x \in G$, that is $[u a, 2 v b]=1$ for all $u, v \in\left\langle x^{G}\right\rangle$ and all $a, b \in L(G)$. The other direction follows trivially from Theorem $\mathrm{E}$.

By $[11]$, we have $L(G)=\varepsilon_{2,1}^{*}(G)$, where

$$
\varepsilon_{2,1}^{*}(G)=\{a \in G ;[a x, 2 y]=[x, 2 y] \text { for all } x, y \in G\}
$$


the first partial 2-Engel margin. Thus for $a \in L(G)$ we observe that $[u a, 2 v b]=$ $[u, 2 v b]$. The usual commutator expansion and observing that $c\left(\left\langle x^{G}\right\rangle\right)=2$, as well as Lemma 5 (i), yield

$$
\begin{aligned}
{\left[u,,_{2} v b\right] } & =[u, b, v b]^{[u, v]^{b}}\left[[u, v]^{b}, v b\right] \\
& =[u, 2 b] \cdot[u, b, v]^{b} \cdot\left[[u, v]^{b}, b\right] \cdot[[u, v], v]^{b} \\
& =([u, b, v][u, v, b])^{b}
\end{aligned}
$$

Thus we arrive at

$$
[u a, 2 v b]=([u, b, v][u, v, b])^{b}
$$

Let $u=\prod_{i=1}^{n} x^{\delta_{i} g_{i}}$ and $v=\prod_{i=1}^{m} x^{\varepsilon_{i} h_{i}}$ with $\delta_{i}, \varepsilon_{i}= \pm 1$ and $g_{i}, h_{i} \in G$. Observing that $[u, b, v] \in Z\left(\left\langle x^{G}\right\rangle\right)$, we obtain by linear expansion that $[u, b, v]$ is a product of commutators of the form $\left[x^{ \pm g}, b, x^{ \pm h}\right]$ with $g, h \in G$. Without loss of generality it suffices to show that $\left[x^{g}, b, x\right]=1$ for $x, g \in G, b \in L(G)$. But this is an immediate consequence of Lemma 5 (ii). It follows that each of the factors in the expansion of $[u, b, v]$ equals 1. A similar argument shows $[u, v, b]=1$. By (1) we conclude that $[u a, 2 v b]=1$.

The following lemma is needed for the verification of certain properties of the counterexample in Proposition 8.

Lemma 7. Let $G$ be a group of class 3 and $a \in L(G)$. Then $a \in \varepsilon_{2}^{*}(G)$ if and only if $[a, x, y]^{3}=1$ for all $x, y \in G$.

Proof: By linear expansion and observing Lemma 5 (i), we obtain

$$
[x, 2 a y]=[x, y, a][x, a, y][x, 2 y] \text {. }
$$

Using $1=[a, 2 x y]=[a, x, y][a, y, x]$ together with the Jacobi identity yields

$$
[x, y, a]=[a, x, y]^{-2}
$$

This together with (2) implies

$$
[x, 2 a y]=[a, x, y]^{-3}[x, 2 y]
$$

If $[a, x, y]^{3}=1$, it follows that $[x, 2 a y]=[x, 2 y]$. By $[11]$, we have already $[x a, 2 y]=$ $[x, 2 y]$ for all $x, y \in G, a \in L(G)$. Hence $a \in \varepsilon_{2}^{*}(G)$. Conversely, if $a \in \varepsilon_{2}^{*}(G)$, we obtain $[a, x, y]^{3}=1$ by (3). 


\section{Proofs of The TheOREMS}

Proof of Theorem 1: Suppose $G=\bigcup_{i=1}^{n} H_{i}$ with $H_{i}$ a 2-Engel group. By Theorem $\mathrm{D}$, we can assume that $\left[G: H_{i}\right]<\infty$ for all $H_{i}$ in the covering. Thus $[G: D]<\infty$ for $D=\bigcap_{i=1}^{n} H_{i}$. Let $h \in D$ and $g \in G$. Since $g \in H_{i}$ for some $i$ and $D \subseteq H_{i}$, we have $[h, g, g]=1$, as $H_{i}$ is a 2-Engel group. Thus $D \subseteq L(G)$, and hence $G / L(G)$ is finite.

Conversely, assume that $G / L(G)$ is finite. Consider $[y, x, x]$ with $y=g^{i} a, x=g^{j} b$ for $g \in G$ and $a, b \in L(G), i, j \in \mathbf{Z}$. Observing again that $L(G)=\varepsilon_{2,1}^{*}(G)$, we have

$$
[y, x, x]=\left[g^{i}, x, x\right]=\left[g^{i}, b, x\right] \text {. }
$$

Further expansion and use of Lemma 5 (iii) and (i) yield

$$
\left[\left[g^{i}, b\right], g^{j} b\right]=\left[g^{i}, b, b\right]\left([[g, b], g]^{b}\right)^{i j}=1 .
$$

We conclude that $[y, 2 x]=1$ for all $y, x \in\langle g\rangle L(G)$. Thus $\langle g\rangle L(G)$ is a 2-Engel group.

Choose a transversal $T=\left\{g_{1}, \ldots, g_{n}\right\}$ of $L(G)$ in $G$. Then $G=\bigcup_{i=1}^{n}\left\langle g_{i}\right\rangle L(G)$, since each $g \in G$ can be written as $g=g_{i} w$ for some $w \in L(G)$ and some. $g_{i} \in T$. By the albove, it follows that $G$ has a covering by finitely many 2-Engel subgroups.

Proof of Theorem 2: Let $G=\bigcup_{i=1}^{n} N_{i}$ with $N_{i}$ a normal 2-Engel subgroup of $G$. It follows immediately by Theorem 1 that $G / L(G)$ is finite. For every $x \in G$ there exists an $N_{i}$ in the normal covering of $G$ with $\left\langle x^{G}\right\rangle \subseteq N_{i}$. Hence every normal closure $\left\langle x^{G}\right\rangle$ is 2-Engel as a subgroup of a 2-Engel group $N_{i}$. It follows by Theorem $E$ that $G$ is a 3-Engel group.

Conversely, assume that $G$ is a 3-Engel group and that $G / L(G)$ is finite. Corollary 6 implies that $\left\langle x^{G}\right\rangle L(G)$ is a normal 2-Engel subgroup for every $x$ in $G$. Choose a transversal $T=\left\{g_{1}, \ldots, g_{n}\right\}$ of $L(G)$ in $G$. Since each $g \in G$ can be written as $g_{i} w$ for some $w \in L(G)$ and some $g_{i} \in T$ we obtain that $G=\bigcup_{i=1}^{n}\left\langle g_{i}^{G}\right\rangle L(G)$. By the above, it follows that $G$ has a covering by finitely many normal 2-Engel subgroups.

Proof of Theorem 3: For $g \in G$ let $H_{g}=\langle g\rangle \psi_{V}^{*}(G)$. Every element in $H_{g}$ can be written as $g^{i} a$ with $a \in \psi_{V}^{*}(G)$ and $i \in \mathbb{Z}$. Let $\psi\left(x_{1}, \ldots, x_{n}\right) \in V$. For $a_{j} \in \psi_{V}^{*}(G), i_{j} \in \mathbf{Z}, j=1, \ldots, n$, we have $\psi\left(g^{i_{1}} a_{1}, \ldots, g^{i_{n}} a_{n}\right)=\psi\left(g^{i_{1}}, \ldots, g^{i_{n}}\right)=1$, since $\langle g\rangle \in \mathcal{V}$. Thus $H_{g} \in \mathcal{V}$ for all $g$ in $G$.

Choose a transversal $T=\left\{g_{1}, \ldots, g_{m}\right\}$ of $\psi_{V}^{*}(G)$ in $G$. Then $G=\bigcup_{j=1}^{m}\left\langle g_{j}\right\rangle \cdot \psi_{v}^{*}(G)$, since each $g \in G$ can be written as $g_{j} a$ for some $a \in \psi_{V}^{*}(G)$ and some $g_{j} \in T$. By the above, each $\left\langle g_{j}\right\rangle \psi_{V}^{*}(G)$ is in $\mathcal{V}$. Thus $G$ has a finite covering by subgroups in $\mathcal{V}$. 


\section{A Counterexample}

For every prime $p$ a $p$-group $H$ of class 3 is constructed whose relevant properties are stated in Proposition 8. The construction of $H$ follows the usual practice. Starting from a group isomorphic to the commutator subgroup of $H$, we will reach $H$ by three split extensions.

Construction of the Counterexample. Let $E_{p}$ denote an elementary abelian $p$-group of countable rank, and $T_{9}$ the direct sum of a countable number of cyclic groups of order 9 . Set $V=\left\langle v_{1}, v_{2}, \ldots\right\rangle, W=\left\langle w_{1}, w_{2}, \ldots\right\rangle$ and $Z=\left\langle z_{1}, z_{2}, \ldots\right\rangle$ with

$$
V \cong W \cong Z \cong \begin{cases}E_{p} & \text { for } p \neq 3 \\ T_{\vartheta} & \text { for } p=3\end{cases}
$$

Define $X=\langle u\rangle \times V \times W \times Z$ with $\langle u\rangle \cong C_{p}$ for $p \neq 3$, and $\langle u\rangle \cong C_{9}$ for $p=3$.

Let $A=[X]\langle a\rangle$, the semidirect product of $X$ with a cyclic group $\langle a\rangle$, where $\langle a\rangle \cong C_{p}$ for $p \neq 3$, and $\langle a\rangle \cong C_{9}$ for $p=3$. The automorplism induced by $a$ on $X$ has order $p$, or 9 , respectively. The action of $a$ on the generators of $X$ is given as follows:

$$
\begin{cases}{[u, a]=1,\left[w_{i}, a\right]=z_{i}^{-1},} & i=1,2, \ldots \\ {\left[v_{i}, a\right]=\left[z_{i}, a\right]=1,} & i=1,2, \ldots\end{cases}
$$

The defining relations of $A$ are those of $X,(4)$, and $a^{p}=1$ for $p \neq 3$, or $a^{9}=1$ for $p=3$, respectively.

Similarly, let $B=[A]\langle b\rangle$ with

$$
\langle b\rangle \cong \begin{cases}C_{p} & \text { for } p \neq 3 \\ C_{9} & \text { for } p=3\end{cases}
$$

where $b$ induces an automorphism of order $p$ or 9 , respectively, on $A$. The action of $b$ on the generators of $A$ is given as follows:

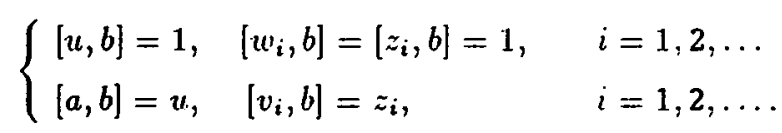

The defining relations of $B$ are those of $A,(5)$, and $b^{p}=1$ for $p \neq 3$, or $b^{9}=1$ for $p=3$, respectively. 
For the last extension we set

$$
C=\left\langle c_{1}, c_{2}, \ldots\right\rangle \cong \begin{cases}E_{p} & \text { for } p \neq 3 \\ T_{\theta} & \text { for } p=3\end{cases}
$$

Let $H=[B] \cdot C$. The elements of $C$ induce automorphisms of order $p$ for $p \neq 3$, or order 9 for $p=3$.

The action of the generators of $C$ on the generators of $B$ is given as follows:

$$
\left\{\begin{array}{lr}
{\left[b, c_{i}\right]=w_{i}, \quad\left[a, c_{i}\right]=v_{i}, \quad\left[u, c_{i}\right]=z_{i}^{2},} & i=1,2, \ldots, \\
{\left[v_{j}, c_{i}\right]=\left[w_{j}, c_{i}\right]=\left[z_{j}, c_{i}\right]=1,} & i, j=1,2, \ldots
\end{array}\right.
$$

The defining relations of $H$ are those of $B,(6)$ and those of $C$. This concludes the construction of $H$.

For the notation in Proposition 8 we refer to that used in the construction of $H$.

Proposition 8. Let $H$ be the group constructed above. Then:

(i) $H=\langle a, b, c\rangle$ and $c(H)=3$, precisely.

(ii) $\exp H= \begin{cases}p & \text { for } p \geqslant 5 \text {, } \\ p^{2} & \text { for } p=2,3\end{cases}$

(iii) $L(H)= \begin{cases}C \cdot H^{\prime} & \text { for } p \neq 3 ， \\ \left\langle a^{3}, b^{3}, C \cdot H^{\prime}\right\rangle & \text { for } p=3 ;\end{cases}$

(iv) $H / L(H)$ is finite, and $H$ is the union of finitely many (normal) 2-Engel subgroups;

(v) $H$ is the union of finitely many (normal) class 2 subgroups;

(vi) $H / \varepsilon_{2}^{*}(H)$ and $H / Z_{2}(H)$ are not finite;

(vii) $\varepsilon_{2}(H)$ is not finite.

Proof: The verification of (i) and (ii) is straightforward and will be omitted here.

(iii) Let $h=a^{\alpha} b^{\beta} c \cdot x \in H$ with $c \in C, x \in X, \alpha, \beta \in Z$. The relations of $H$ together with $c(H)=3$ imply that

$$
\left[c_{i}, h, h\right]=\left(\left[c_{i}, a, b\right]\left[c_{i}, b, a\right]\right)^{\alpha \beta}=\left(z_{i} z_{i}^{-1}\right)^{-\alpha \beta}=1 .
$$

Hence $C \subseteq L(H)$. Since $H^{\prime} \subseteq L(H)$ because of $c(H)=3$, it follows that $C H^{\prime} \subseteq$ $L(H)$.

To establish the rest of the claim, let $x=a^{\alpha} b^{\beta} c h^{\prime}$ with $c \in C, h^{\prime} \in H^{\prime}$, and $\alpha, \beta \in \mathbf{Z}$. Using the relations of $H$, we obtain for $s, t \in \mathbf{Z}$

$$
\left[a^{*} b^{\ell}, x, x\right]=z^{3(\beta s-\alpha t)} \text { for some } z \in Z \text {. }
$$


Let $p=3$. If $s=3$ and $t=0$, or $s=0$ and $t=3$, we have $\left[a^{a} b^{t}, x, x\right]=1$ for all $x \in H$. Hence $a^{3}, b^{3} \in L(H)$ in this case.

Conversely, for any $p$, let $0 \leqslant s \leqslant p-1,0 \leqslant t \leqslant p-1$, and set $c=c_{1}$. Then (7) becomes

$$
\left[a^{s} b^{t}, x, x\right]=z_{1}^{3(\beta,-\alpha t)} .
$$

It can be easily seen that for given $s$ and $t$, not both equal to zero, there exist integers $\alpha, \beta$ such that $z_{1}^{3(\beta s-\alpha t)} \neq 1$, hence $a^{s} b^{\ell} \notin L(H)$ in this case. It follows that $L(H) \subseteq C H^{\prime}$ for $p \neq 3$, and $L(H) \subseteq\left\langle a^{3}, b^{3}, C H^{\prime}\right\rangle$ for $p=3$.

(iv) The finiteness of $H / L(H)$ is an inmediate consequence of (iii). The rest follows by Theorem 1, Theorem 2 , and the fact that $c(H)=3$.

(v) First let $p \neq 3$. The 2-Engel group $\langle g\rangle L(H), g \in H$, is of nilpotency class 2 . Thus the finite covering by 2-Engel groups resulting from Theorem 1 is a finite covering by (normal) sulggroups of class 2 .

This is not the case if $p=3$, for example for $a, b^{3}, c_{1} \in\langle a\rangle L(H)$ we have $\left[a, b^{3}, c_{1}\right]=z_{1}^{6} \neq 1$. Thus $c(\langle a\rangle L(H))>2$. However, for $p=3$ we will show that $H=\bigcup_{0 \leqslant s, t \leqslant 8}\left\langle a^{0} b^{t}\right\rangle C \cdot H^{\prime}$ is a finite covering by (normal) subgroups of class 2.

Set $g=a^{s} b^{t}, 0 \leqslant s, t \leqslant 8$, and let $y_{1}, y_{2}, y_{3} \in C H^{\prime}$. Using $C H^{\prime} \subseteq L(H)$ and $c(H)=3$, we obtain by linear expansion

$$
\left[g^{i} y_{1}, \dot{g}^{j} y_{2}, g^{k} y_{3}\right]=\left[g, y_{2}, y_{3}\right]^{i}\left[y_{1}, g, y_{3}\right]^{j}
$$

Using the relations of $H$, we obtain $\left[y_{1}, g\right]=g_{1}$ and $\left[g, y_{2}\right]=g_{2}$ with $g_{1}, g_{2} \in$ $V \times W \times Z$. Hence $\left[g, y_{2}, y_{3}\right]=\left[y_{1}, g, y_{3}\right]=1$. This together with (8) leads to $\left[g^{i} y_{1}, g^{j} y_{2}, g^{k} y_{3}\right]=1$. Thus we may conclude that the above finite covering consists of subgroups of class 2 .

(vi) We will show $c_{i} \not \equiv c_{j} \bmod \varepsilon_{2}^{*}(H)$ for $i \neq j$. By Lemma 7 , it suffices to establish $\left[c_{i} c_{j}^{-1}, a, b\right]^{3} \neq 1$. Using the relations of $H$, we obtain

$$
\left[c_{i} c_{j}^{-1}, a, b\right]^{3}=\left[c_{i}, a, b\right]^{3}\left[c_{j}, a, b\right]^{-3}=z_{i}^{-3} z_{j}^{3} \neq 1 .
$$

Thus there exist infinitely many different cosets $\bmod \varepsilon_{2}^{*}(H)$ in $H$. Since $Z_{2}(H) \subseteq$ $\varepsilon_{2}^{*}(H)$ by [11], we have that $H / Z_{2}(H)$ is not finite either.

(vii) We observe that $\left[a, b c_{i}, b c_{i}\right]=z_{i}^{3} \neq 1$. Thus $Z^{3} \subseteq \varepsilon_{2}(H)$. Since $Z^{3}$ is not finite, the claim follows.

\section{REFERENCES}

[1] M.A. Brodie, R.F. Chamberlain and L.C. Kappe, 'Finite coverings by normal subgroups', Proc. Amer. Math. Soc. (to appear). 
[2] P. Hall, 'Verbal and Marginal Subgroups', J. Reine Angew. Math. 182 (1940), 156-157.

[3] L.C. Kappe and W.P. Kappe, 'On three-Engel groups', Bull; Austral. Math. Soc. 7 (1972), $391-405$.

[4] W.P. Kappe, 'Die A-Norm einer Gruppe', Illinois J. Math. 5 (1961), 187-197.

[5] I.D. Macdonald, 'Some Examples in the Theory of Groups', Mathematical Essays Dedicated to A.J. MacIntyre, pp. 263-269 (Ohio University Press, 1970).

[6] B.H, Neumann, 'Groups covered by permutable subsets', J. London Math Soc. 28 (1954), 236-248.

[7] B.H. Neumann, 'Groups covered by finitely many cosets', Publ. Math. Debrecen 3 (1954), $227-242$.

[8] H. Neumann, Varieties of groups, Ergeb. Math. Grenzgb. 37 (Springer Verlag, Berlin, 1967).

[0] D.J.S. Robinson, Finiteness conditions and generalized soluble groups $I$, Ergeb. Math. Grenzgb. 62 (Springer Verlag, Berlin, 1972).

[10] J. Schur, 'Über die Darstellungen der endlichen Gruppen durch gebrochene lineare Substitutionen', J. Reine Angew. Math. 127 (1904), 20-30.

[11] T.K. Teague, 'On the Engel Margin', Pacific J. Math. 60 (1974), 205-214.

Department of Mathẹmatical Sciences

University Center at Binghamton

State University of New York

Binghamton, N.Y. 13901

United States of America 